\title{
Distribución, tamaño y estructura poblacional de Jubaea chilensis en "Las Palmas", comuna de Petorca, región de Valparaíso - Chile
}

\author{
Jubaea chilensis distribution in "Las Palmas", \\ Borough of Petorca, Region of Valparaíso - Chile
}

\section{Cristian Youlton a*, Cristina Hormazabal a, Ignacio Schiappacasse a, Patricia Contreras a, Carlos Poblete-Echeverría ${ }^{a}$}

\begin{abstract}
*Autor de correspondencia: a Pontificia Universidad Católica de Valparaíso, Escuela de Agronomía, Quillota, Chile, casilla 4D, Quillota, Chile, tel.: +56-32-2274508, cristian.youlton@pucv.cl
\end{abstract}

\section{SUMMARY}

Chilean palms (Jubaea chilensis) are endemic of central Chile and are classified as vulnerable according to the category of conservation. Even though the population of palms has gone down, there are still some groups (palmar) that remain in locations such as Petorca, Chile. In Petorca, the site "Las Palmas" was declared "Priority Site for the Biodiversity Conservation". However, the number of palms, their age and spatial distribution in "Las Palmas" are still unknown. Thus, in order to describe the conservation status, a population survey of palms was done. Location and age of every palm (infantile, child, adult or old, according to morphological features) were noted and their position georeferenced. Data were analyzed using a geographic information system that included maps and satellite imagery (Quickbird II). To assess the spatial distribution of palms, a Complete Spatial Randomness (CSR) test was applied using an L-function. Main results showed that 1,300 palms are present in "Las Palmas". Among those palms, $41 \%$ are infantile, $45 \%$ are young, $14 \%$ are adults and $0.5 \%$ are old plants. Infantile, young and adult palms have an aggregated distribution in high and inaccessible areas of the watershed headwater, while old palms have disaggregated distribution. The palmar is crossed by route E-37-D, where some adult palms that produce seeds are commonly present. However, if any seed gets to infantile palm, it does not reach the juvenile stage. There is no regeneration in lowland and in the proximity of communities. Thus, the palmar is reducing its extension.

Key words: age distribution, palm forest, georeferencing, sclerophyllous forest.

\section{RESUMEN}

La palma chilena (Jubaea chilensis) es una especie endémica de Chile central, clasificada en categoría de conservación vulnerable. De su área de distribución original subsisten algunas agrupaciones (palmares), encontrándose una de ellas en la comuna de Petorca, en el límite norte de la región de Valparaíso. El sector "Las Palmas" fue declarado "Sitio Prioritario para la Conservación de la Biodiversidad", sin embargo, se desconoce el número de palmas presentes, su distribución etaria y espacial. Para caracterizar el estado del palmar "Las Palmas" en Petorca, se realizó un catastro de todos los individuos de Jubaea chilensis presentes. Se registraron las coordenadas y edad de cada ejemplar, datos que fueron representados en un SIG complementado con cartografía e imagen satelital Quickbird II. Su distribución espacial se analizó mediante la función L mediante una prueba CSR (Completed Spatial Randomness). Como resultado del catastro, se registraron 1.300 ejemplares, $41 \%$ infantiles, $45 \%$ juveniles, $14 \%$ adultas, y $0,5 \%$ de viejas. Infantiles, juveniles y adultos presentan distribución agregada en sectores altos e inaccesibles en la cabecera de la subcuenca, mientras que palmas viejas están dispersas en el área. El palmar es atravesado por la ruta E-37-D, en sus alrededores se registran palmas adultas que producen semillas, sin embargo, muchas de ellas son extraídas para consumo humano, y las que llegan a emerger, no alcanzan el estado juvenil. No existe regeneración en sectores bajos y próximos a áreas pobladas. De esta forma, el palmar está sufriendo la reducción de su superficie.

Palabras clave: distribución etaria, palmar, georeferenciación, bosque esclerófilo.

\section{INTRODUCCIÓN}

La palma chilena (Jubaea chilensis (Mol.) Baillon), es una especie endémica de Chile continental y monoespecífica para su género. Es una planta perenne con un tronco de hasta $2 \mathrm{~m}$ de diámetro, puede alcanzar $35 \mathrm{~m}$ de alto y vivir más de 400 años de edad. Su estado infantil se caracteriza por el crecimiento de hojas a ras de suelo hasta que la planta alcanza su diámetro basal definitivo, para luego crecer en altura mediante el desarrollo del tronco en el estado juvenil. Alcanza la madurez reproductiva y fructifica a partir de los 35-70 años de edad, adelgazando el diámetro de crecimiento del tronco en altura, adquiriendo una figura similar a una botella (Grau 2004).

La especie se distribuye en el centro del país, en áreas costeras entre el río Limarí (subcuenca de Quebrada Culi- 
mo) y Tapihue, región del Maule (Michea 1993), asociado con bosque matorral esclerófilo desde el nivel del mar hasta los $700 \mathrm{~m}$ de altitud. Esta zona del país también se caracteriza por concentrar la mayor población urbana y rural, por lo que el paisaje se encuentra altamente intervenido y bajo constante presión por la expansión agrícola y urbana (Schulz et al. 2010), de su infraestructura asociada (carreteras, gasoductos, líneas de transmisión eléctricas), deforestación por incendios, explotación de leña o pastoreo (Quintanilla y Castillo 2007, 2009, Flores-Toro y AguirreSaavedra 2008, Quintanilla y Morales 2013). Debido a la alta modificación del hábitat original, y al alto endemismo de especies, esta zona es considerada un punto caliente (hot-spot) de biodiversidad a nivel mundial, prioritario para su conservación (Myers et al. 2000). La disminución o desaparición del matorral en el hábitat de la palma afecta su regeneración, ya que la especie requiere la protección de una cobertura vegetal nodriza en su estado infantil, y ante su inexistencia queda expuesta a la predación de lagomorfos y ganado doméstico (Fleury et al. 2015). La regeneración de la especie in situ también se ve interrumpida debido a la fuerte explotación directa de sus semillas para consumo directo humano. Debido a todo lo anterior, esta especie fue declarada protegida en el territorio nacional (Decreto 908 de 1941), en estado de conservación "vulnerable" según la Lista de Especies Amenazadas de UICN (González 1998).

Actualmente los bosques o "palmares" existentes son relictos de su distribución original, siendo los más conocidos los de Ocoa (Parque Nacional La Campana en la región de Valparaíso) con más de 70.308 ejemplares, seguido de Cocalán (Parque Nacional y terrenos privados en la región de O'Higgins) con 35.500 individuos, y en tercer lugar con 7.200 ejemplares adultos el palmar de Siete Hermanas, al sur oriente de la ciudad de Viña del Mar, región de Valparaíso. Los dos primeros palmares poseen un nivel de protección y manejo que permite asegurar su renovación, en tanto que el último presenta una población envejecida sin regeneración. A nivel nacional, los ejemplares de la especie no superarían los 120.000 individuos (González et al. 2009).

En el límite entre las regiones de Valparaíso y Coquimbo se encuentra la cuesta "Las Palmas", con uno de los palmares relictos más septentrionales del país, donde su población y distribución etaria es desconocida. El área fue declarada Sitio Prioritario para la Conservación de la Biodiversidad a nivel nacional (Muñoz et al. 1996); sin embargo, se desconoce el número de individuos que componen el palmar y su estado de conservación. El presente estudio tiene como objetivo determinar la distribución espacial y etaria de individuos de Jubaea chilensis mediante un catastro poblacional del palmar "Las Palmas" de Petorca.

\section{MÉTODOS}

Área de estudio. El palmar "Las Palmas" se ubica en el norte de la región de Valparaíso (figura 1), comuna de Petorca (coordenadas UTM 19H 296765 E - 6438726 S, datum WGS 1984). Lo atraviesa la ruta E-37-D, cuyo trazado correspondía a la antigua línea del tren al norte, construida en 1910.
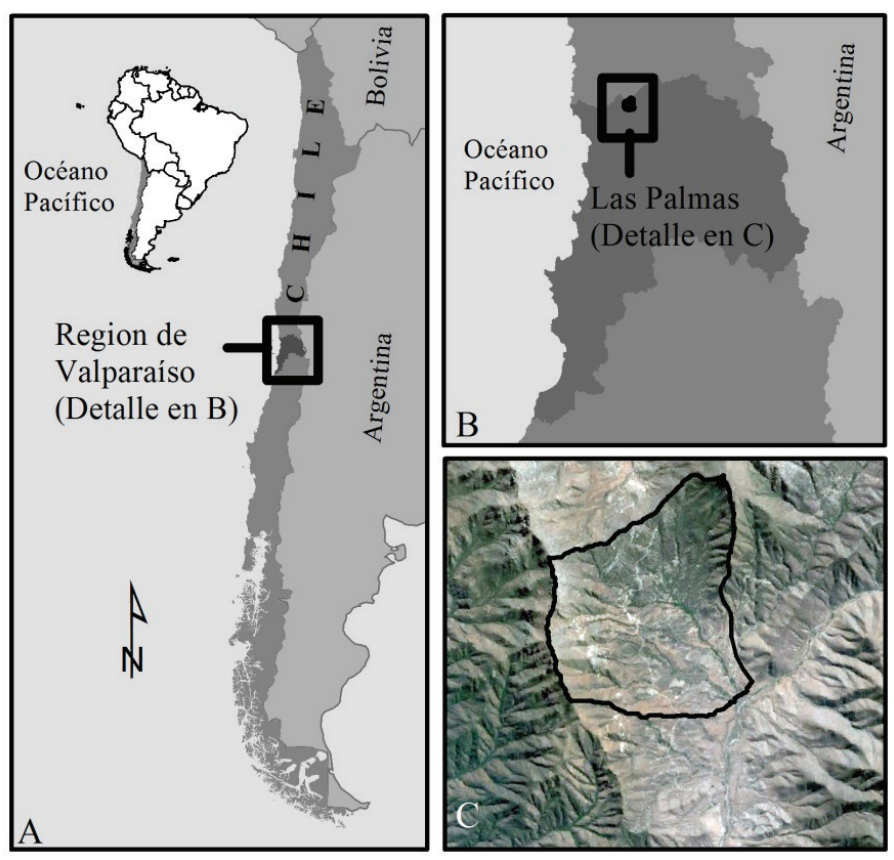

Figura 1. Ubicación del área de estudio, en el límite norte de la Región de Valparaíso. C) Cabecera de la subcuenca del estero Las Palmas.

Location of the study area at the northern edge of Valparaiso Region. (C) Shows a watershed headwater of Estero Las Palmas. 
El catastro se realizó en la cabecera del estero Las Palmas, tributario del río Petorca, que escurre de norte a sur. La zona estudiada tiene $6 \mathrm{~km}$ de largo por $4 \mathrm{~km}$ de ancho (en promedio), con una superficie de $24 \mathrm{~km}^{2}$. El punto más alto está a 1.775 m s.n.m. (cerro Maray), en tanto que el más bajo a 500 m s.n.m. En el sector bajo de relieve ondulado (con pendiente $<30 \%$, bajo la cota $800 \mathrm{~m}$ s.n.m.) se ubica el poblado de Las Palmas, con 108 habitantes, en tanto que en la zona alta (relieve de lomajes, cerros y montañas con pendiente $>30 \%$ ), junto a la cuesta está El Manzano, con 46 habitantes (datos levantados el año 2006). Las actividades económicas desarrolladas en el sector son minería (sector alto de los cerros, mayoritariamente por habitantes de El Manzano), agricultura y ganadería (lomajes en la zona baja, por habitantes del junto al poblado de Las Palmas).

Según Luebert y Pliscoff (2006), el área de estudio se encuentra en el piso vegetacional de bosque esclerófilo mediterráneo costero de Cryptocarya alba (Mol.) Looser y Peumus boldus Mol. En sectores de exposición sur se encontró Quillaja saponaria (Mol.), Cryptocarya alba, Retanilla trinervia (Gillies et Hook.) Hook. et Arn. y Lithraea caustica (Mol.) H. et A. En sectores soleados y rocosos se encontró Colliguaja odorifera Mol., Adesmia microphylla Hook. et Arn., Alonsoa meridionales (L.F.) O.Kunze, Retanilla trinervia, Puya berteroniana Mez. y Colletia hystrix Clos. En fondos de quebradas se encontró Drimys winteri J.R. et Forster y Luma chequen (Mol.) A. Gray, acompañados de Azara petiolaris (D.Don) I.M.Johnst., Aristotelia chilensis (Mol.) Stuntz y Maytenus boaria Mol. En términos generales, la vegetación se encuentra más perturbada hacia sectores bajos y próximos a poblados.

Catastro de palmas. Entre junio y noviembre del 2006 se recorrió a pie toda el área de estudio, geo-referenciando todos los individuos de palma con un GPS marca Garmin, modelo Summit. Para representar la distribución de palmas en el área de estudio se empleó el software ArcMap 9 empleando como base cartográfica la carta IGM 1:50.000 (Tilama). Se utilizó la imagen satelital Quickbird II (con resolución de $0,6 \mathrm{~m}$ en la banda pancromática) capturada el 22 de marzo del 2006 para fotointerpretar aspectos de interés del terreno (sectores poblados, deforestados, caminos secundarios, etc.). Las palmas visualizadas en sectores de difícil acceso se identificaron espacialmente en la imagen satelital.

Para cada palma se determinó su edad según las siguientes características (Michea 1988): i) infantil, sólo presenta desarrollo radial de su copa a ras de suelo; ii) juvenil, con crecimiento vertical por desarrollo del tronco; iii) adulto, presenta desarrollo reproductivo, evidenciado por la presencia de racimos de semillas en su copa y adelgazamiento del tronco en su porción superior.; y iv) viejo, donde la fracción adelgazada de su tronco es proporcionalmente mayoritaria. Según Hacienda Las Palmas de Cocalán (2010), dueña del palmar del mismo nombre, las palmas infantiles tienen $0-20$ años de edad, juveniles en- tre 20 y 60 años, adultas desde 60 años y viejas pueden alcanzar más de 20 a $30 \mathrm{~m}$ de altura a los 1.000 años de edad.

Análisis de la distribución espacial de las palmas. Con las coordenadas de la ubicación geográfica de las palmas se realizó una prueba CSR (Completed Spatial Randomness) mediante la función L que corresponde a la versión normalizada de la K de Ripley, con intervalos de confianza al $95 \%$ generados mediante la simulación de Monte Carlo con 1.000 repeticiones. La prueba de CSR se sustenta en la asunción de que un conjunto de datos mapeados, para un área determinada, seguirá el patrón definido por una distribución de Poisson homogénea, es decir, tendrá una distribución al azar (Diggle 2003). De modo que, si dichos individuos se distribuyesen de forma agregada, dado un individuo cualquiera, la probabilidad de encontrar a un individuo diferente sería menor que si tuviesen una distribución aleatoria. Utilizando esta función en la prueba de CSR se puede conocer si la distribución de la muestra es agregada, aleatoria o regular (Diggle 2003). El análisis espacial de la distribución de las palmas fue realizado con el software estadístico R (versión 3.2.5) utilizando el módulo spatstat (Baddeley y Turner 2005).

\section{RESULTADOS}

Se identificaron 1.300 individuos de palma. La mayor proporción correspondió a individuos juveniles (580 ejemplares, $45 \%$ ), seguido de infantiles (531 individuos, 41 $\%)$. Apenas 183 palmas (14\%) son adultas, responsables del aporte de semillas para la regeneración del palmar y seis palmas $(0,5 \%)$ fueron identificadas como viejas.

A nivel general, el palmar se ubica en la cabecera de la subcuenca, en su límite norte (figura 2). Los individuos infantiles se ubican junto al camino (64 palmas), pero en mayor número por sobre este (369 plantas), en un sector de difícil acceso protegido por acantilados graníticos. Otro núcleo de 98 individuos infantiles crecen en el sector nororiente, asociados a la presencia de matorral cerrado en un sector de difícil acceso. Entre ambos centros existe un camino minero abandonado que facilita el ingreso y pastoreo de ganado (principalmente caprino), el que consume las plántulas, modifica la composición y densidad del matorral, y finalmente impide la renovación del palmar.

Ya el estado juvenil presenta el mayor número de plantas y se distribuye, al igual que los individuos infantiles, en dos sectores: i) mayoritariamente (447 palmas) por sobre el camino, próximo al límite norte de la subcuenca; y ii) en menor número (130 ejemplares) sobre laderas del sector nor-oriente. Los únicos individuos juveniles que se encuentran en el sector poblado fueron plantados en la escuela rural de "Las Palmas".

Las palmas adultas son las que mostraron la mayor distribución en el territorio, concentrándose 164 plantas en los sectores altos. Se encuentran algunos individuos adultos (19 palmas) en los sectores bajos de la subcuenca, 
A. Palmas Infantiles

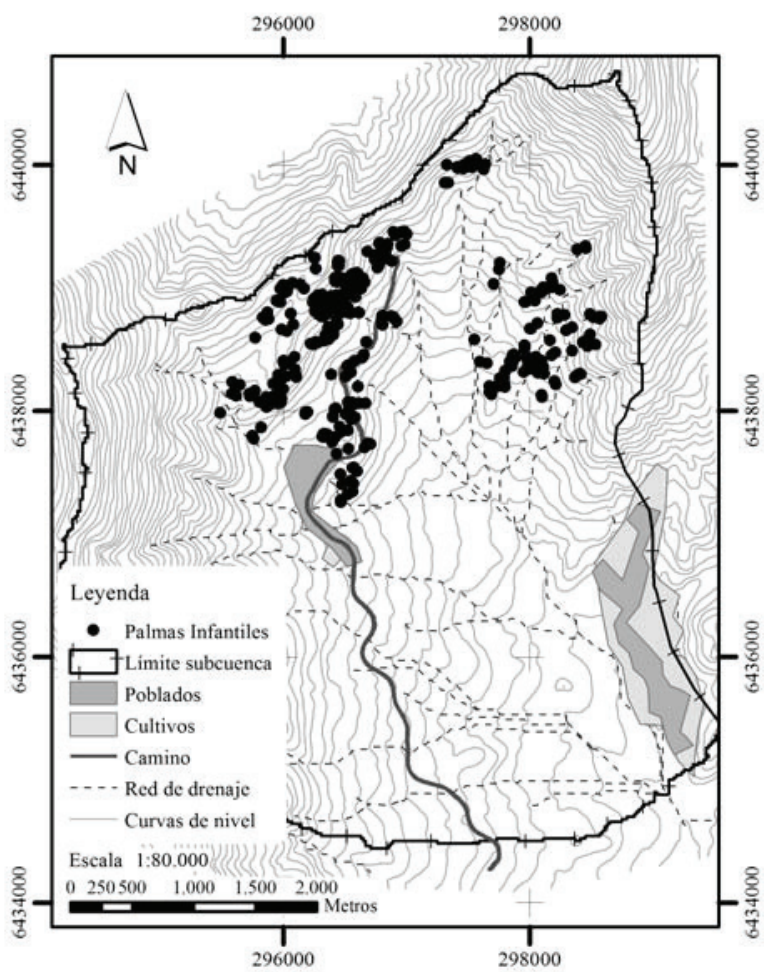

C. Palmas Adultas

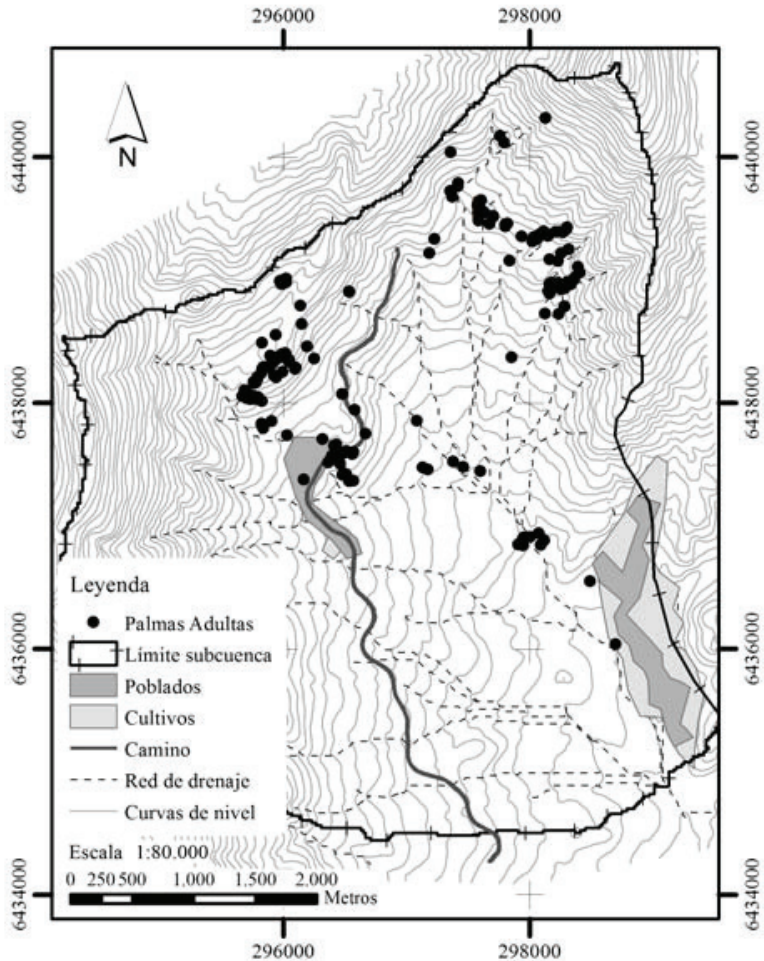

B. Palmas Juveniles

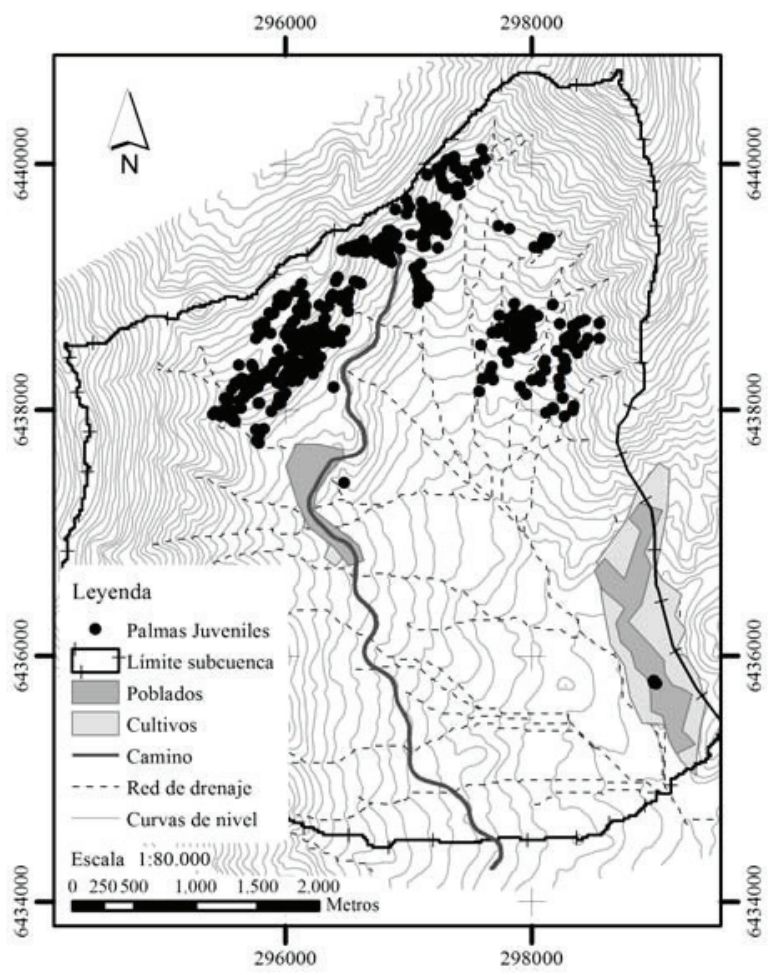

D. Palmas Viejas

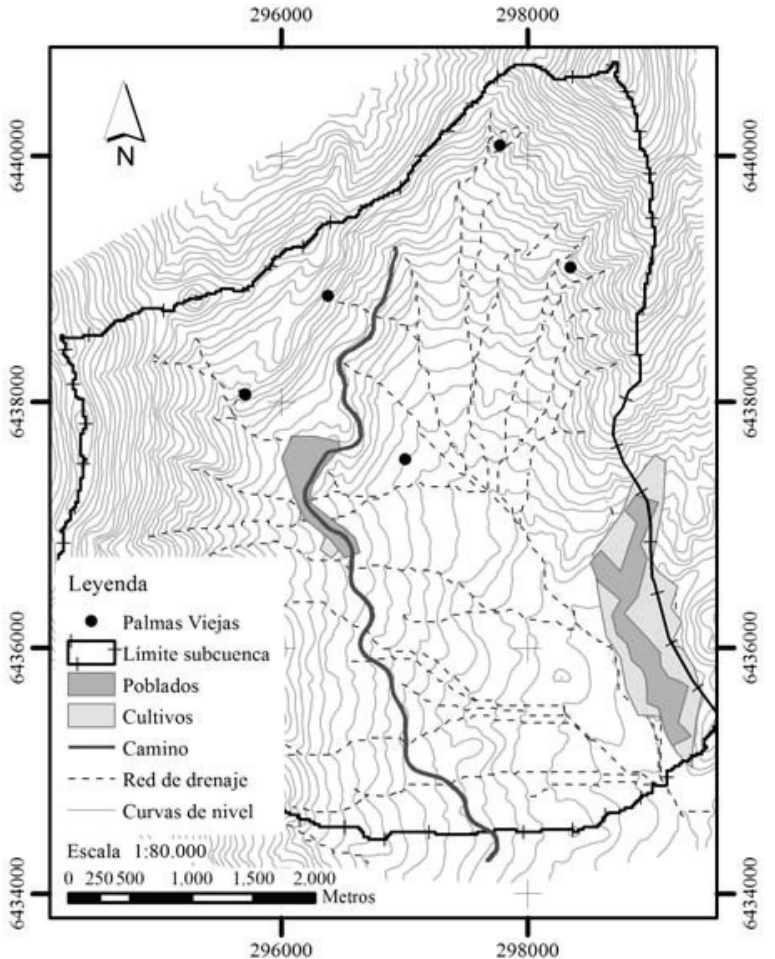

Figura 2. Distribución de palmas en el área de estudio, por edad. Cada punto indica una palma individual.

Age distribution of palms in the study area. Each point represents an individual palm. A) Infantile palms. B) Young palms. C) Adult palms. D) Old palms. 
próximos a los cursos de agua (donde la vegetación es más densa) y sectores poblados. El área de lomajes cercana a lugares poblados se encuentra altamente erosionado debido a su uso agropecuario actual y pasado, por lo que presenta un paisaje altamente intervenido, con 120 hectáreas de suelos con escasa cobertura herbácea y alta presión de ganado, de las cuales 3,8 hectáreas son surcos y cárcavas. Por este motivo, el sector bajo no presenta renovación de palmas, siendo los actuales individuos adultos remanentes recientes de la distribución original del palmar. Respecto de los individuos viejos, solo existen seis individuos en sectores alejados y de difícil acceso.

En la figura 3 se puede observar que, para los casos de palmas infantiles, juveniles y adultas, la curva de la función L(r)-r empírica se encuentra fuera del espacio definido por los intervalos de confianza obtenidos en la simulación de patrones CSR. En estos casos la función L(r)-r presenta valores mayores a cero, lo que indica la existencia de una fuerte agregación. Para el caso de la distribución de palmas infantiles el valor máximo de la función L(r)-r, alcanzó un valor de alrededor de $800 \mathrm{~m}$ mientras que para palmas juveniles y adultas los valores máximos de la función fueron de alrededor de 700 y $500 \mathrm{~m}$, respectivamente. Para las palmas viejas, la función L(r)-r presentó valores mayores a cero (agregación) hasta una distancia $r$ de alrededor de 650 metros y para distancias superiores el valor de L(r)-r fue menor a cero indicando la presencia de dispersión. Cabe destacar que, en este caso, existen solo seis observaciones lo que implica la presencia de un sesgo impórtate en el análisis de la función $\mathrm{L}$.

\section{DISCUSIÓN}

En el área de estudio destaca la inexistencia de individuos infantiles y juveniles fuera de la cabecera norte de la subcuenca de Las Palmas. Esto sería explicado por la modificación del hábitat causado por la extracción de leña y el uso agrícola de suelos frágiles, principalmente en el área de lomajes cercanos al poblado de Las Palmas. Evidencia de ello es la presencia de amplios sectores deforestados
A. Infantiles

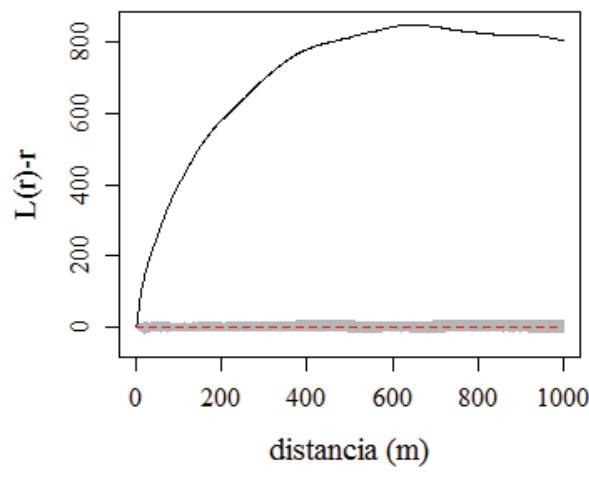

C. Adultas

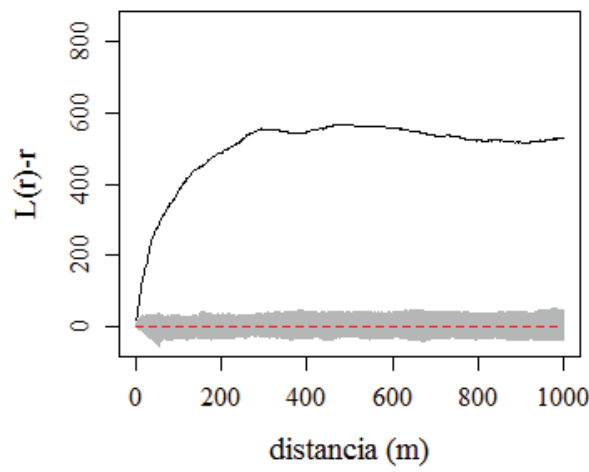

B. Juveniles

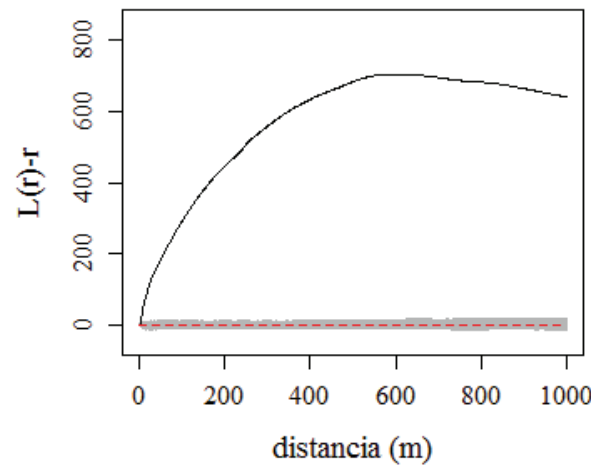

D. Viejas

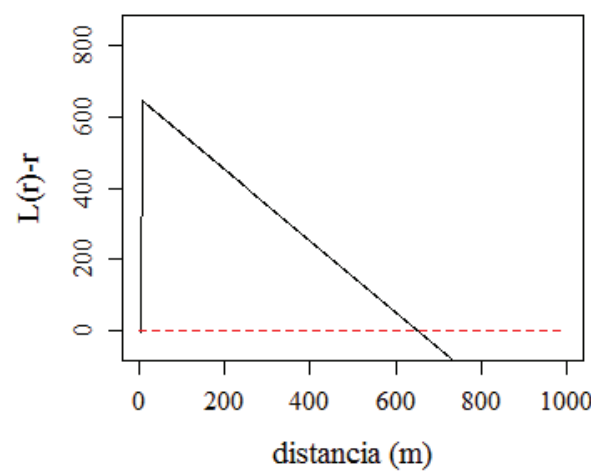

Figura 3. Resultados de la función de $K$ Ripley normalizada (L(r)-r), por edad de las palmas. La línea discontinua indica el valor esperado de una distribución aleatoria, la línea sólida representa la función L(r)-r y el área sombreada muestra los intervalos de confianza al $95 \%$ generados con la simulación de Monte Carlo.

Results of Ripley's K function normalized (L(r)-r) by age. The dashed line is the expected value for a random distribution, the solid line depicts the L(r)-r function and the gray area showed $95 \%$ confidence intervals generated by Monte Carlo simulation. 
y fuertemente erosionados, agravados por el actual pastoreo del ganado caprino y bovino que ramonea las hojas de las plántulas de palmas y arbustos asociados (Michea 1988, Marcelo et al. 2006, MMA 2007). Además se suma la predación ejercida por especies introducidas (conejos) y nativos (degú, Octodon degus Mol.), (Marcelo et al. 2006, Fleury et al. 2015). A pesar de que en las áreas bajas hay palmas adultas que aportan semillas, no hay regeneración del palmar, por lo que su extensión se reducirá en el futuro.

A la predación del ganado sobre plántulas de palmas, debe sumarse la extracción de sus semillas para consumo humano directo (González et al. 2009). Los lugareños señalan la extracción furtiva y organizada de coquitos (como se conoce a sus semillas) por personas foráneas, lo que se evidencia al encontrar numerosos racimos cortados y las herramientas utilizadas, especialmente a orilla del camino. A pesar de ello, se encuentran palmas infantiles próximas a la ruta (64 palmas), no así individuos juveniles. Esto indica que la extracción de semillas no es absoluta en los alrededores de la vía, sin embargo, las plantas que logran emerger no sobrepasan el estado infantil, probablemente por predación del ganado caprino y bovino que accede fácilmente desde el camino. Tampoco puede descartarse la eventual extracción de palmas pequeñas a orilla del camino por parte de visitantes ocasionales.

Jubaea chilensis es una especie que requiere de una cobertura arbustiva que actúe como nodriza (Fleury et al. 2015). Esta condición favorable para la regeneración del palmar está acotada a zonas de difícil acceso en la cabecera de la subcuenca, lo que concuerda con la distribución agregada calculada (análisis (L(r)-r)) de palmas infantiles, juveniles y adultas. Esta agregación a zonas de difícil acceso es similar a la descrita por Michea (1988) en el palmar de Ocoa. Si bien la mayoría de palmas infantiles y juveniles se ubican en sectores altos que presentan individuos productores de semillas, llama la atención que justo por sobre el túnel hay escasos adultos, muchos juveniles y algunos infantiles. Esto podría ser explicado por una posible extracción de palmas adultas 20 a 30 años antes, permaneciendo su descendencia en el lugar. A pesar de ser una especie reconocidamente longeva, llama la atención la casi inexistencia de palmas viejas (seis individuos), ubicadas en sectores alejados y de muy difícil acceso. Esto sería explicado por el relato de lugareños que señalan una gran extracción en la década de 1940.

La actual renovación del palmar depende de 183 plantas reproductivamente activas, sin embargo, sólo se registra descendencia apenas de 164 palmas (12\%) que se ubican en el sector alto. El número de individuos infantiles pudo ser subestimado debido al pequeño tamaño de plántulas que quedan ocultas en sectores de matorral denso. El número de palmas infantiles es menor que la población juvenil, indicando que en los últimos 20 a 30 años se ha producido un aumento de la predación de semillas y plántulas, o bien la modificación del hábitat que desfavorece su implantación. De continuar esta situación, la renovación del palmar se verá amenazada y limitada sólo a sectores de difícil acceso, lo que ya ha sido reportado en otros palmares de la zona central de Chile (González et al. 2009).

\section{CONCLUSIONES}

A pesar de que el palmar "Las Palmas" está alejado de centros urbanos y presenta una topografía escarpada que limita su acceso, su conservación presenta amenazas por la modificación y reducción del hábitat. La población local de Jubaea chilensis presenta dominancia de individuos juveniles por sobre infantiles y adultos, los que tienen distribución agregada. Los pocos individuos viejos remanentes poseen una distribución desagregada en el área de estudio. De continuar esta tendencia, se iniciará un proceso de envejecimiento del palmar. La regeneración de palmas se da solamente en áreas restringidas y de difícil acceso en la zona alta de la subcuenca. No existe renovación en sectores bajos donde hay palmas adultas, por lo que el palmar está condenado a reducir su tamaño. Al año 2006, el palmar declarado Sitio Prioritario para la Conservación estaba constituido por 1.300 individuos.

\section{AGRADECIMIENTOS}

El catastro se realizó en el marco del proyecto "Ordenamiento territorial del sector "Las Palmas" de Petorca, para el uso sustentable de un palmar relicto", financiado por el Fondo de Protección Ambiental de la Comisión Nacional del Medio Ambiente (FPA-CONAMA, Código 05049-06), 2006. Los autores agradecen a la comunidad de Las Palmas por el apoyo e información brindada para la ejecución del proyecto, y a los alumnos del curso de Conservación de Recursos Naturales de la carrera de Agronomía PUCV que apoyaron las campañas de terreno.

\section{REFERENCIAS}

Baddeley A, Turner R. 2005. Spatstat: an R package for analyzing spatial point patterns. The Journal of Statistical Software 12:1-42.

Diggle PJ. 2003. Statistical analysis of spatial point patterns. London, UK. Edward Arnold. 159 p.

Fleury M, W Marcelo, RA Vásquez, LA González, RO Bustamante. 2015. Recruitment Dynamics of the Relict Palm, Jubaea chilensis: Intricate and Pervasive Effects of Invasive Herbivores and Nurse Shrubs in Central Chile. PLoS ONE 10:e0133559.

Flores-Toro L, F Aguirre-Saavedra. 2008. Floral richness of the Nature Sanctuary Palmar El Salto, Viña del Mar, Valparaiso region, Chile. Gayana Botanica 65:71-83.

González L, R Bustamante, C Navarro, M Herrera, M Toral Ibañez. 2009. Ecology and management of the Chilean palm (Jubaea chilensis): history, current situation and perspectives. Palms 53:68-74.

González M. 1998. Jubaea chilensis. Consultado 08 ene. 2016. Disponible en http://dx.doi.org/10.2305/IUCN.UK.1998. 


\section{RLTS.T38586A10128158.en}

Grau J. 2004. Palmeras de Chile. Santiago, Chile. Oikos. 203 p. Hacienda Las Palmas de Cocalán. 2010. Palma chilena, Generosa y renovable. Consultado 5 de febrero 2016. Disponible en http://www.cocalan.cl/2010/docs/LA\%20PALMA\%20 CHILENA.pdf

Luebert F, P Pliscoff. 2006. Sinopsis bioclimática y vegetacional de Chile. Santiago, Chile. Editorial Universitaria. 316 p.

Marcelo W, R Bustamante, R Vásquez. 2006. Efectos de la herbivoría, el microhábitat y el tamaño de las semillas en la sobrevivencia y crecimiento de plántulas de la palma chilena. Ambiente y Desarrollo 22:55-62.

Michea G. 1988. Estudio poblacional de la palma chilena (Jubaea chilensis) en el sector de Ocoa, Parque Nacional La Campana. Medio Ambiente 9:124-130.

Michea G. 1993. Las palmeras chilenas: historia de sobrevivencia. Chile Forestal 204:32-33.

MMA. 2007. Ficha de antecedentes de especie. Jubaea chilensis. Consultado 10 mar. 2016. Disponible en http://www.mma. gob.cl/clasificacionespecies/Anexo_tercer_proceso/plantas/Jubaea_chilensis_FINAL.pdf

Muñoz M, H Núñez, J Yáñez. 1996. Libro rojo de los sitios prioritarios para la conservación de la diversidad biológica en
Chile. Santiago, Chile. Ministerio de Agricultura, Corporación Nacional Forestal. 203 p.

Myers N, RA Mittermeler, CG Mittermeler, GAB Da Fonseca, J Kent. 2000. Biodiversity hotspots for conservation priorities. Nature 403:853-858.

Quintanilla V, M Castillo. 2007. Study of the populations of Chilean palm affected by fire in the hills and gorges of the Colinaje coast in the fifth region, Central Chile. Revista Geografica de Chile Terra Australis 51-52:165-176.

Quintanilla V, M Castillo. 2009. Degradación de ecosistemas de la palma más austral del mundo (Jubaea Chilensis) acelerada por los fuegos estivales en los cordones litorales de Valparaíso y Viña del Mar (32'50'-33” 02's). Un caso sostenido de perturbación del paisaje. Investigaciones Geográficas 41:41-60.

Quintanilla V, M Morales. 2013. Disturbances of the fires of summer in the palm more southern of world (Jubaea Chilensis (mol.) Baillon) in coastal microbasins of the zone Mediterranean of Chile. Cuadernos Geográficos 52:129-152.

Schulz JJ, L Cayuela, C Echeverria, J Salas, JM Rey Benayas. 2010. Monitoring land cover change of the dryland forest landscape of Central Chile (1975-2008). Applied Geography 30:436-447. 
